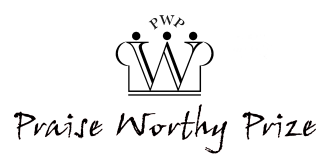

\title{
Optimization Techniques for Prognostics of On-Board Electromechanical Servomechanisms Affected by Progressive Faults
}

\author{
Matteo D. L. Dalla Vedova, Pier Carlo Berri
}

\begin{abstract}
In relatively recent years, electromechanical actuators have gradually replaced systems based on hydraulic power for flight control applications. Electromechanical servosystems are typically operated by electrical machines that transfer rotational power to the controlled elements (e.g. the aerodynamic control surfaces) by means of gearings and mechanical transmission. Compared to electrohydraulic systems, electromechanical actuators offer several advantages, such as reduced weight, simplified maintenance and complete elimination of contaminant, flammable or polluting hydraulic fluids. On-board actuators are often safety critical; then, the practice of monitoring and analyzing the system response through electrical acquisitions, with the aim of estimating fault evolution, has gradually become an essential task of the system engineering. For this purpose, a new discipline, called Prognostics, has been developed in recent years. Its aim is to study methodologies and algorithms capable of identifying such failures and foresee the moment when a particular component loses functionality and is no longer able to meet the desired performance. In this paper, authors have introduced the use of optimization techniques in prognostic methods (e.g. model-based parametric estimation algorithms) and have proposed a new model-based fault detection and identification method, based on Genetic Algorithms optimization approach, able to perform an early identification of the aforesaid progressive failures, investigating its ability to identify timely symptoms alerting that a component is degrading. Copyright (C) 2019 The Authors.

Published by Praise Worthy Prize S.r.l. This article is open access published under the CC BY-NC-ND license (http://creativecommons.org/licenses/by-nc-nd/3.0/).
\end{abstract}

Keywords: EMA, Fault Detection/Identification (FDI), GA, Model-Based, Prognostics

\section{Nomenclature}

ACE

ADC

ANN

BLDC

$d t$

EHA

EMA

EMF

$e_{i}$

err

$e_{\text {tot }}$

$f\left(\theta_{m}\right)$

FDI

GA

$I_{\text {ref }}$

$I_{m}$

$I_{r}$

$\boldsymbol{k}$

$K_{\text {emf }}$

$\mathrm{MM}$

$N_{a, b, c}$

$\mathrm{NC}$

$P$

$\begin{array}{ll}\text { PID } & \text { Proportional Integrative Derivative } \\ \text { PHM } & \text { Prognostics and Health Management } \\ \text { PWM } & \text { Pulse Width Modulation } \\ \text { RM } & \text { Reference Model } \\ \text { RUL } & \text { Remaining Useful Life } \\ \text { RVDT } & \text { Rotary Variable Differential Transformer } \\ T R & \text { Load torque } \\ Z & \text { Normalized amplitude of rotor eccentricity } \\ \theta_{m} & \text { Rotor angular position } \\ \zeta & \text { Amplitude of static rotor eccentricity } \\ \phi & \text { Phase of static rotor eccentricity }\end{array}$

\section{Introduction}

In aeronautical applications, actuation systems based on the electromechanical technology (EMAs) are progressively replacing the well-known hydraulic systems, starting from the less safety critical uses (e.g. the actuation of trim tabs, cargo bay doors, or weapon and sensor systems of military aircraft), up to the most important ones, like primary and secondary flight controls [1]. EMAs are typically operated by electrical machines that transfer mechanical power to the controlled elements (e.g. aerodynamic surfaces) through gearings and mechanical transmission. 
Compared to the more traditional electrohydraulic systems, they offer several advantages, mainly related to the absence of a centralized hydraulic power generation and distribution system.

In this way, despite the lower power per unit weight characteristic of electrical machines compared to hydraulic ones, an overall weight reduction can be achieved by eliminating the systems and the components related to hydraulic power generation and distribution. In addition, maintenance costs can be reduced, since training of maintenance personnel for servicing hydraulic components is not needed anymore; moreover, the maintenance interventions on electric subsystems are intrinsically easier, since they do not require to deal with hydraulic fluid, which is usually pollutant, degradable, flammable or easily affected by contamination [2]-[4].

The EMA technology is quite recent and the reliability of these systems is not yet adequately known; then, dedicated diagnostic strategies (usually based on redundancy, scheduled maintenance and periodic inspections) shall be employed in order to guarantee a suitable risk reduction. In fact, given that actuators for aerospace applications are often safety critical, it is necessary to create redundancies and schedule maintenance operations able to guarantee the system to operate always in safety conditions. Those strategies, in spite of their many merits and benefits, unavoidably imply a significant cost increase and sometimes limit the diffusion of the electromechanical actuation systems. In particular, a failure mode peculiar of electromechanical systems (and virtually inexhistent in hydraulic actuators) is the mechanical jamming of the transmission. This failure mode has the effect of locking the affected flight control in the current position, making the aircraft incontrollable; the action of a parallel backup actuator is mostly ineffective. Strategies that can mitigate this risk include the use of series backup actuators or velocity sum devices, which greatly increase the total system cost and weight [5], [6].

Additionally, these methods of risk reduction are often ineffective against failures caused by unexpected and extreme scenarios, such as anomalous operative conditions exceeding the aircraft flight envelope. Even if currently they are the most common strategies to reduce the risks, they can turn out unsuccessful in these unpredictable situations, thus requiring unscheduled maintenance interventions.

For example, the operation of a component outside its envelope can result in exceeding the design loads, causing an initial damage (i.e. the beginning of a crack in a structural or mechanical component, a local thermal damage on electrical equipment, or a dent in the rolling surface of a bearing). Then, if the damage remains undetected because of its limited extent and limited effect on the system performance, normal operation of the vehicle may cause the fault to progressively grow, up to hindering the system to work within its functional and performance requirements. A well known and documented case of this kind of damage propagation is the metal structures fatigue on [7]-[10].

However, similar behaviors can be found in a multitude of other fault modes.

In this regard, in recent years, a new engineering discipline, called Prognostics and Health Management (PHM), has been developed as an innovative strategy to reduce risks associated with the propagation of progressive failures [11]. PHM relies on the continuous monitoring of functional parameters of the system in order to detect and identify the precursors of failures at an early stage, in order to estimate the Remaining Useful Life (RUL) of the components [12]. This information about the healt condition of a system and its subsystems can be leveraged in maintenance planning. As a result, most of the necessary maintenance interventions can be scheduled ahead instedad of being performed as corrective maintenance; the operation profile of the aircraft can be adaptedively modified in order to reduce ground time, resulting in higher availability and lower operating costs. In addition, The adoption of a reliable prognostic strategy supporting the aircraft maintenance activity will ultimately lead to a simpler troubleshooting task, reducing the total ground time of the vehicle and mitigating the risks associated with human factor in fault identification [13].

In case of EMAs, PHM can be applied in a more efficient way than in the case of hydromechanical or electrohydraulic actuators, because, on electrical systems, additional sensors are not required, since several informative quantities are already measured for control purposes.

In fact, the application of the PHM strategies normally entails the monitoring of a set of parameters in the form of electric signals, often sharing the same sensors of the control scheme and system monitors [14]. The monitored parameters are usually converted into electrical signals in order to perform these prognostic analyses, so a PHM approach is particularly convenient when applied to an electromechanical system, where most parameters are already in form of electric signals without the need for dedicated sensors and transducers. This avoids increasing the overall costs and worsening the system basic reliability; in addition, a prognostic strategy that does not require invasive hardware modifications is easier to retrofit on existing systems, allowing a faster application on field

The first step of PHM analysis consists in the Fault Detection and Identification (FDI) phase [15]. At this stage of the prognostic task, the system response is analyzed in order to identify the early signs of incipient faults. The detected system fault condition is then leveraged to inform the subsequent RUL estimation phase.

Several different approaches are proposed in literature, either best suited for offline or real-time fault detection, based on their accuracy and computational cost. A primary classification of FDI strategies distinguishes between model-based and data-driven techniques. 


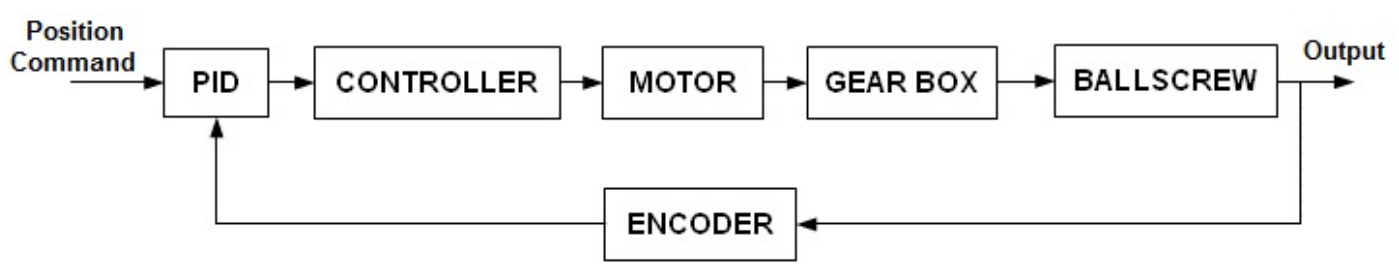

Fig. 1. Conceptual scheme of the considered electromechanical actuator [37]

Model Based FDI relies on the comparison of the system output with the one of a simulation model for the health monitoring, either in the time domain [12], [16][23] or leveraging spectral analysis [24]-[29] and Fast Fourier Transform [30], [31]. These techniques usually feature a high accuracy for the fault detection, but they require a deep knowledge of the physical behavior of the system, not only regarding the response in Nominal Conditions (NC), but also in presence of multiple failure modes, in order to develop reliable and accurate high fidelity models of the system. Moreover, the abovementioned models are often associated to a very high computational cost, with evaluation times ranging from several seconds to several days, which makes them unsuitable for the real-time execurtion with the constrained hardware and the limited processing power available onboard the vehicle; in some extreme cases, these strategies may also become impractical for offline use during the maintenance activity, since a traditional, manual troubleshooting of the faulty system may result quicker. Conversely, Data-Driven approaches to FDI leverage a large data base of system output in place of an exhaustive knowledge of the physical system; data is used to train machine learning tools employed to estimate the health status of the monitored equipment. Different methods are found in literature, such as Artificial Neural Networks (ANNs) [32]-[36] or Fuzzy Logic [37],[38]. These techniques are usually characterized by a reduced computational cost in evaluation, which makes them best suited for real-time health monitoring. However, they require a computationally intensive training phase, often performed offline, and their accuracy is typically lower than Model-Based techniques. The effectiveness of a Data-Driven FDI method is strictly related to the statistical representativeness and the completeness of the training data base, as well as the employed training strategy. A sufficiently complete training dataset is seldom available from field data, albeit possibly easier to obtain than high fidelity models of the system able to simulate the presence of multiple failure modes.

A further group of FDI strategies [15], [39]-[43] exploits a combination of the aforementioned methods in a hybrid approach. In this way, they try to leverage the advantages of both Model-Based and Data-Driven FDI. For example, high fidelity models can be used to gather the training dataset, while machine learning tools work as fast surrogate models in order to speed up the computations online and achieve real-time performances.

However, most of the FDI strategies found in literature are suitable for the isolation of a single fault mode out of a small number of possible choices. In order to achieve a robust fault detection, able to support the maintenance activity of a complex system such as an aircraft, the ability to reliably isolate and identify a large number of multiple failure modes is needed.

\section{I.1. Brief Summary of the Proposed FDI Approach}

In this work, the use of optimization techniques in prognostic methods is introduced (e.g. model-based parametric estimation algorithms) and a new modelbased FDI method, based on the Genetic Algorithm (GA) optimization, able to perform an early identification of the previously mentioned progressive failures is proposed, investigating its ability to timely identify symptoms alerting that a component is degrading. For this purpose, the authors have considered a typical EMA layout (Fig. 1) according to the "More-electric-aircraft" paradigm proposed by Quigley [1] and the "All-electricaircraft" paradigm shown by Howse [2]. Operationally speaking, the proposed FDI method is divided into three sequential phases:

- comparing the dynamic response of the real actuator (affected by several possible combinations of progressive failures) with a corresponding monitoring system (a simplified numerical model simulating the behavior of the monitored EMA);

- using an optimization process, implemented by a GA [16], [17] that minimizes the value of proper objective functions by acting on defined parameters of the model;

- correlating the obtained parameters (of the aforesaid monitoring model) with the actual magnitudes of the considered EMA progressive failures.

In order to provide a versatile test bench on which evaluate the dynamic response of the actuator in different fault conditions, authors have developed a detailed numerical model (simulating the effects of several progressive faults) that has been assumed as a representative of the actual physical system [45].

Different combinations of progressive faults have been considered in order to evaluate the accuracy of the prediction at the different conditions and to assess the field of validity of the proposed method. In particular, five progressive faults have been considered, namely dry friction torque and backlash affecting the EMA mechanical transmission, turn to turn short circuit affecting the coils of the three stator phases of the electric motor, rotor eccentricity and drift of the PID controller proportional gain. 
In this manuscript, Section II describes in detail the electromechanical system considered in our work and the related numerical models, Section III illustrates the setup of the optimization problem and Section IV discusses the results of the investigation. In Section $\mathrm{V}$ potential applications of this works are proposed, as well as future possible developments.

\section{Electromechanical Actuator Layout}

Until a few years ago, the actuators used mainly in aeronautical applications have been generally hydraulic and in particular hydro-mechanical or, more recently, electro-hydraulic. This type of actuators, due to their great precision, high specific power and high reliability, often equip current aircraft (even if, on last generation airliners, electro-hydrostatic actuators (EHA) or electromechanical actuators (EMA) have been installed). In recent years, the trend towards fully electric aircraft has led to a wide application of new optimized electric actuators, such as the electromechanical ones. As shown in Fig. 2, a typical EMA used in a primary flight control is composed by:

- an actuator control electronics (ACE) that closes the feedback loop, by comparing the commanded position with the actual one, elaborates the corrective actions and generates the reference current $I_{r e f}$;

- an electrical motor, often BLDC (BrushLess Direct Current) type;

- a gear reducer having the function to decrease the motor angular speed and increase its torque to desired values;

- a system that transforms rotary motion into linear motion: ball screws or roller screws are usually preferred to ACME screws because, having a higher efficiency, they can perform the conversion with lower friction;

- a network of sensors used to close the feedback rings (current, angular speed and position) that control the whole actuation system (reported in Figure 2 as RVDT).

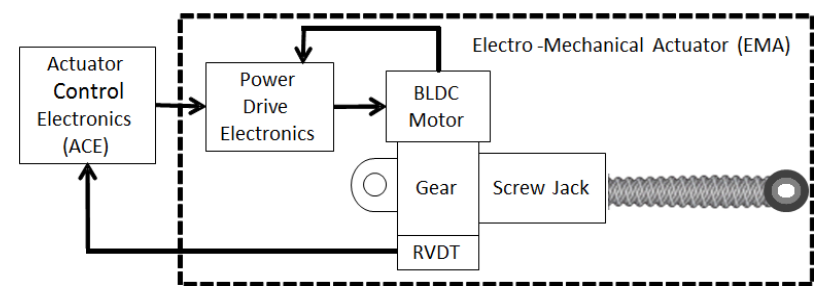

Fig. 2. Schematic of the considered electromechanical actuator [44]

\section{II.1. EMA Numerical Models}

As already mentioned, two numerical models with different complexity and fidelity of the considered EMA are used for this research, both developed in the MATLAB-Simulink simulation environment.

A low fidelity model, referred to as the Monitor Model (MM), features a low computing time and is suitable for the iterative execution needed during the optimization algorithm, which essentially consists in the search of the global minimum of a function of eight variables. A high fidelity model, referred to as the Reference Model (RM), is employed as a simulated test bench for the validation of the Monitor Model and the FDI algorithm as a whole. The RM architecture, widely described in [35] and [39], is represented in the EMA block diagram shown in Fig. 3.

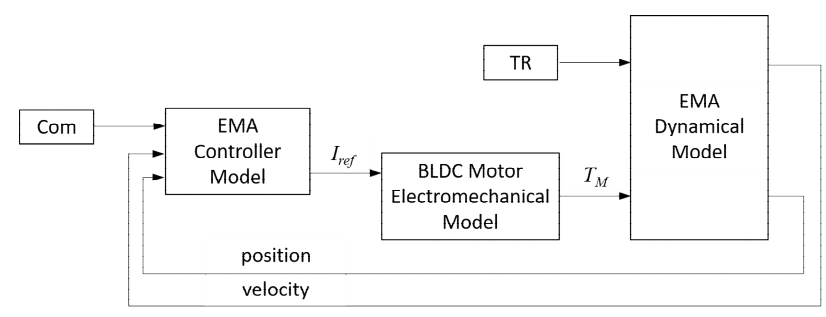

Fig. 3. EMA reference model (RM) block diagram

The main subsystems of the EMA model are briefly described in the following:

- Com: generates input position commands.

- EMA Controller Model: simulates the actuator control electronics, closing the position and speed feedback loops and computing as output the reference current $I_{r e f}$. This is not a physical current, but rather the digital input of the power drive electronics, which applies a current equal to $I_{\text {ref }}$ to the BLDC stator phases through a hysteresis control.

- BLDC Motor ElectroMechanical Model: simulates the power drive electronics through a SimScape model and the trapezoidal BLDC EM behavior, evaluating torque as a function of three-phase current generated by an ideal $\mathrm{H}$-bridge regulator.

- EMA Dynamic Model: resolves the dynamic equation of mechanical behavior by a two D.o.F. dynamic system.

- TR: input block simulating the aerodynamic torques acting on the moving surface controlled by the actuator.

This numerical model simulates the behavior of the real EMA taking also into account the effects of BLDC motor non-linearities, such as partial phase short circuit an rotor eccentricity [46]-[51], end-of-travels, compliance and backlashes acting on the mechanical transmission [52], [53], ADC conversion of the feedback signals, electrical noise acting on the signal lines and electrical offset of the position transducers [15] and dry friction (e.g. acting on bearings, gears, hinges and screw actuators) [54].

The RM performs a very detailed simulation of the functional behavior of the system, even in presence of various combinations of fault modes, but features the main disadvantage of being computationally heavy: a medium performance laptop can run this model taking more than one minute for each second of simulation, or about two orders of magnitude above real time simulation. 


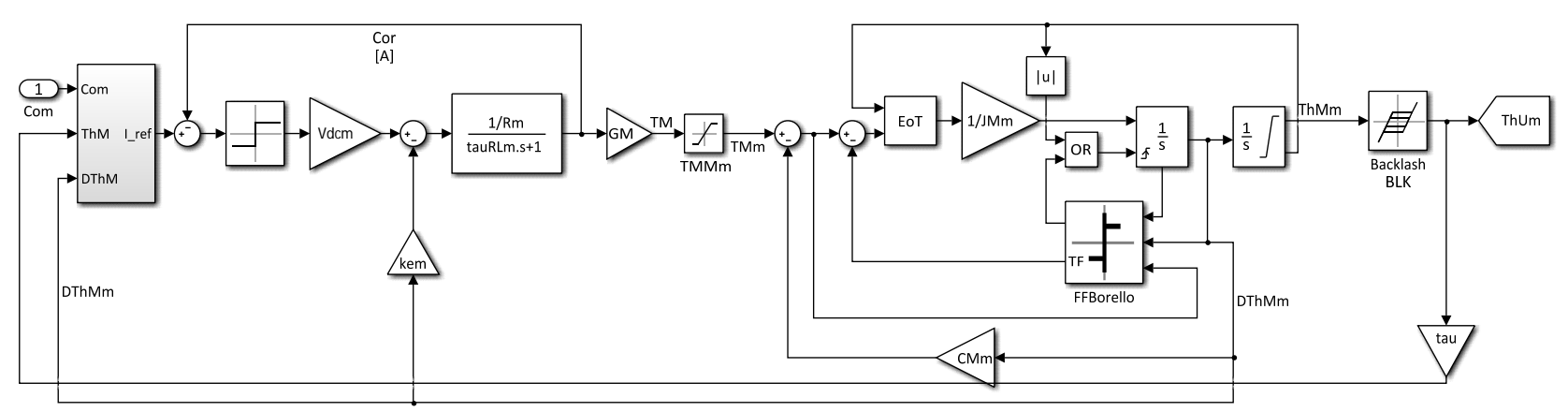

Fig. 4. EMA monitoring model (MM) block diagram [14]

This is not acceptable if the model is to be used in an iterative on-line computation, requiring a computing time not compatible with the on field use of an FDI algorithm for prognostic purposes during scheduled maintenance or preflight tests. For this reason, a lower fidelity model is developed as a simplified representation of the considered EMA, yet it is detailed enough to simulate the effect of all the considered faults with high accuracy. The architecture of the MM is represented in Fig. 4 (and similar to that proposed in [14]). The main simplification of the MM is the elimination of the three-phase trapezoidal control of the BLDC, replaced by a much simpler and computationally lighter single-phase equivalent scheme. The complex PWM current regulation is substituted by a simplified hysteresis control, avoiding digital-to-analog conversion of signals and phase switching logic. This solution allows a time step one order of magnitude longer to be employed for the simulation, greatly reducing computing time to almost real-time; however, a complication arises for the simulation of electrical faults, which will be discussed in the following paragraph.

\section{II.2. Modelization of Fault Modes}

For the FDI algorithm to be effective in an on-field application, the greatest possible number of fault modes shall be considered and implemented in the simulations, in order to allow a good matching of the dynamic response between the Monitor Model and the physical system. Five different fault modes have been considered for the study and have been chosen among the most common for EMAs, as highlighted by [55]-[57].

Moreover, they are usually characterized by a progressive evolution, making possible an effective prognostic detection [58]. The considered faults are briefly listed below:

- Dry friction due to the wearing of mechanical components;

- Backlash of the reducer gearbox and/or rotary-tolinear conversion device;

- Partial short circuit of the BLDC stator coils;

- Rotor eccentricity due to the degradation of its support bearings;

- Control electronics fault resulting in the drift of the
PID controller Proportional gain. This can be determined by multiple causes, such as electrical contact deterioration, overtemperature of semiconductor components or position sensor faults.

It should be noticed that the partial short circuit is characterized by three degrees of freedom (corresponding to the fraction of damaged windings of each phase), while the rotor static eccentricity is described by two parameters (i.e. its amplitude $\zeta$ and phase $\phi$, which consist in the angle measured between the minimum air gap and the phase A stator coil). This results in the health condition of the actuator to be described by a vector $\boldsymbol{k}$ of eight elements:

- $\quad k_{1}$ is the normalized friction: $k_{1}=0$ means nominal conditions, while $k_{1}=1$ means $300 \%$ of nominal condition.

- $\quad k_{2}$ is the normalized backlash: $k_{2}=0$ means nominal condition, $k_{2}=1$ means 100 times the nominal condition. Although this range may seem exaggerated at a first glance, 100 times the nominal condition means about half a radian of mechanical play on the fast shaft, reduced by the gear ratio to $5.7 \cdot 10^{-2}$ degrees on the slow shaft or $20 \%$ of the already small chirp command amplitude.

- $k_{3}, k_{4}$ and $k_{5}$ are respectively the normalized short circuit of phases $\mathrm{A}, \mathrm{B}$ and $\mathrm{C}$; for example, $k_{3}=0$ means a fully functional phase $\mathrm{A}$, while $k_{3}=1$ means a complete short circuit for the same phase.

- $\quad k_{6}$ is equal to the eccentricity amplitude parameter $\zeta$.

- $k_{7}$ is the phase of rotor eccentricity $\phi$, i.e. the direction corresponding to the minimum air gap; $k_{7}=$ 0 means $\phi=-180^{\circ}, k_{7}=1$ means $\phi=180^{\circ}$.

- $k_{8}$ is the normalized variation of the proportional gain: $k_{8}=0$ is a $50 \%$ reduction of the proportional gain, while $k_{8}=1$ means a $50 \%$ increase.

The Backlash, Dry Friction and Proportional Gain Drift faults implementation is quite straightforward in both models, requiring only the dynamic adjustment of the corresponding parameters in the RM and MM. However, the simplification introduced in the MM with the elimination of the three-phase electrical model somehow complicates the representation of the Partial Short Circuit and Rotor Eccentricity faults. In the RM, those can be simulated by differentially altering the resistance, inductance and electromagnetic coupling parameters of the three phases. The same cannot be done 
for the MM, since it employs an equivalent single phase computational scheme. This issue has been overcome with the introduction of two non-physical shape functions [14], which modulate the counter electromotive force and stator resistance according to the angular position of the rotor, allowing the MM to reproduce the current waveforms typical of the presence of one of the electrical faults, as shown in Fig. 5. The analysis of the behavior of the RM in presence of the electrical faults has led to the following definition of the shape functions, which have no physical meaning, but allow fitting the behavior of the RM in terms of phase current with satisfying accuracy while being acceptably simple to evaluate:

- The Short Circuit fault is simulated by changing the Stator Resistance and Torque Gain according to the active stator phases, by means of a modulating coefficient $f\left(\theta_{m}\right)$, dependent on the rotor angular position $\theta_{m}$ :

$$
f\left(\theta_{m}\right)= \begin{cases}\frac{N_{b}+N_{c}}{2}, & -\frac{\pi}{6}<\theta_{e}<\frac{\pi}{6} \\ \frac{N_{c}+N_{a}}{2}, & \frac{\pi}{6}<\theta_{e}<\frac{\pi}{2} \\ \frac{N_{a}+N_{b}}{2}, & \frac{\pi}{2}<\theta_{e}<\frac{5}{6} \pi\end{cases}
$$

where $N_{a}, N_{b}, N_{c}$ are the fraction of healthy windings of each stator phase.

- The Rotor eccentricity fault is implemented by modulating the Back-EMF and Torque Gain according to the angular position of the rotor, with a function that matches the waveform produced by the RM:

$$
\begin{aligned}
K_{e m f}= & K_{e m f}^{N C}\left(1-Z\left(\cos \left(P \theta_{m}+\phi\right)+\right.\right. \\
& \left.\left.+\sigma\left(6 P \theta_{m}+\phi\right) \sin \left(P \theta_{m}+\phi\right)\right)\right)
\end{aligned}
$$

where $K_{\text {emf }}$ is the back-EMF constant, $N C$ denotes nominal conditions, $Z$ is the normalized rotor eccentricity, $\phi$ is the eccentricity phase, $P$ is the number of pole pairs, $\theta_{m}$ is the rotor angular position and $\sigma(x)=x / 2 \pi-[x / 2 \pi]$.

\section{Fitness Function and GA Settings}

In the following sections, the optimization algorithm chosen to solve the FDI task is described (Section III.1); then, the optimization problem is described in terms of fitness (i.e. objective) function (Section III.2) and the settings of the solver are summarized (Section III.3).

\section{III.1. Genetic Algorithms: Generalities}

The FDI problem can be seen as the minimization of the error between the stator current curves of the two EMA models, with fixed fault parameters on the RM and varying the ones of the MM; when the error between the two curves approaches zero, the fault parameters of the two models are expected to match. The complexity of the error function, which features a strong non linearity and a high dimensionality of the fault space, leads to the choice of a Genetic Algorithm (GA) for the optimization.

GAs are robust non-deterministic optimization techniques inspired by the Darwinian evolution biological process; they are particularly suited for the optimization of computationally expensive functions with a domain with a high dimensionality.

A Genetic algorithm solves a given problem consisting in optimizing an objective function, which is referred to as the Fitness Function, using a population of solutions which, initially random generated, are made to evolve to a number of successive generations until they converge on a minimum fitness value. In the presented case the fitness function will be dependent on the integral over time of a quadratic error between the responses of the RM and MM.

Considering a chromosome population representing the possible solutions of the problem, the fitness function measures the quality of each solution, i.e. how well the solution fits with the problem: in a sense, it can be seen as the adaptability of an individual to the environment. Individuals with best values of the fitness function have higher probability to transmit their own genes to the next generation; therefore, each generation usually contains better individuals than the previous one, gradually evolving toward the global optimum solution. Being GAs non-deterministic techniques, it is not guaranteed that they can find an accurate global optimal solution, but they are able to find good solutions in a reasonable time. In the traditional model, chromosomes are bit strings of fixed length and all generations have the same number of individuals; each chromosome represents a point in the search space. Two basic operators are employed for producing the next generation of solutions starting from the current one:

- Recombination or Crossover combines genes of two individuals to produce children individuals, providing exploitation of the best solution currently available, trying to make the algorithm converge on local minima;

- Mutation randomly generates new genes reintroducing genetic material lost, thus allowing the exploration of the search space in order to find the global minimum.

\section{III.2. Fitness Function and Test Command}

The fitness function is computed as a quadratic error between the dynamic responses of the RM and MM. The response of the EMA should be considered in terms of a parameter that is both easy to measure in a physical system and visibly affected by a great number of progressive damages, even in their incipient phase. For this reason, the user angular position is not suitable as monitored parameter for prognostic applications, since 
although already measured by the RVDT transducers needed to close the feedback loop, it is altered only slightly by the presence of an incipient fault, which is almost completely compensated by the robustness of the control logic. The best choice is then the motor current, which is visibly influenced by various failure modes even when they do not alter the position response; moreover, the phase current is already measured for closing the hysteresis control loop of the power drive electronics. In order to compare the single phase current of the MM with the three phase RM, the envelope of the three phase currents is computed and filtered in order to cancel signal noise mainly produced by the PWM control and external disturbances.

The error is then evaluated with a total least squares method (as shown in [59]), which consists in numerically integrating the squared normal distance between the two curves. This method is chosen instead of a traditional least squares method since it features better performances in presence of uncertainties on both the measured current and time, especially near step discontinuities in the curves. The resultant fitness function is then:

$$
e r r=d t \cdot \sum_{i} \frac{\left(I_{r(i)}-I_{m(i)}\right)^{2}}{\frac{\left(d I_{r(i)} / d t\right)^{2}}{k}+1}
$$

where $I_{r}$ and $I_{m}$ are the reference and monitor single phase currents and $k$ is a constant used to normalize the derivative of the reference current, chosen as its RMS value, so that the current curves have a unitary mean derivative and the uncertainties in the current and time coordinate have approximately the same effect on the fitness function.

In this way however, the different fidelity of the two models causes a small offset between the correct matching of the fault parameters and the global minimum of the fitness function. In order to reduce this offset, the electrical parameters of the $\mathrm{MM}$ are calibrated to minimize the error in nominal condition (i.e. with no faults affecting the models). Moreover, the residual error in nominal conditions is subtracted to the fitness function, to impose a zero fitness value when both models are set in nominal conditions. The input position command to be applied to both EMA models is chosen as a chirp with amplitude of $5 \times 10^{-3} \mathrm{rad}$ and frequency increasing linearly from zero to $15 \mathrm{~Hz}$ in 0.5 seconds. This command is chosen because it allows spanning a range of frequencies, highlighting different behaviors of the actuator and showing the effect of a high number of failure modes: a step or ramp command would have hidden the effect of some faults, especially the electrical ones. The small amplitude of the input signal, on the other hand, is required in order to reduce the position drift between the two models, which would offset the effect of the electrical faults. In fact, the smallest possible command should be chosen in order to limit the position error between the two models to a fraction of a revolution of the motor shaft. The chosen value of $5 \times 10^{-3}$ rad is the threshold amplitude that can be precisely followed by an EMA with flight grade position sensors, possibly mounted on an intermediate shaft to increase resolution. Another advantage of using a small amplitude test command is the possibility, in future works, to superimpose the test command itself to that generated by the pilot or flight control computer; in this way, if the computing time can be kept acceptably low, the prognostic FDI could be performed even in real time during flight.

\section{III.3. Settings of the GA}

The GA employed for the prognostic optimization is the one implemented in MATLAB Optimization Toolbox; in order to achieve a good convergence, accuracy and robustness of results, some tuning of the algorithm settings is needed. In the following, the optimization algorithm parameters that have been modified from their default value are briefly described.

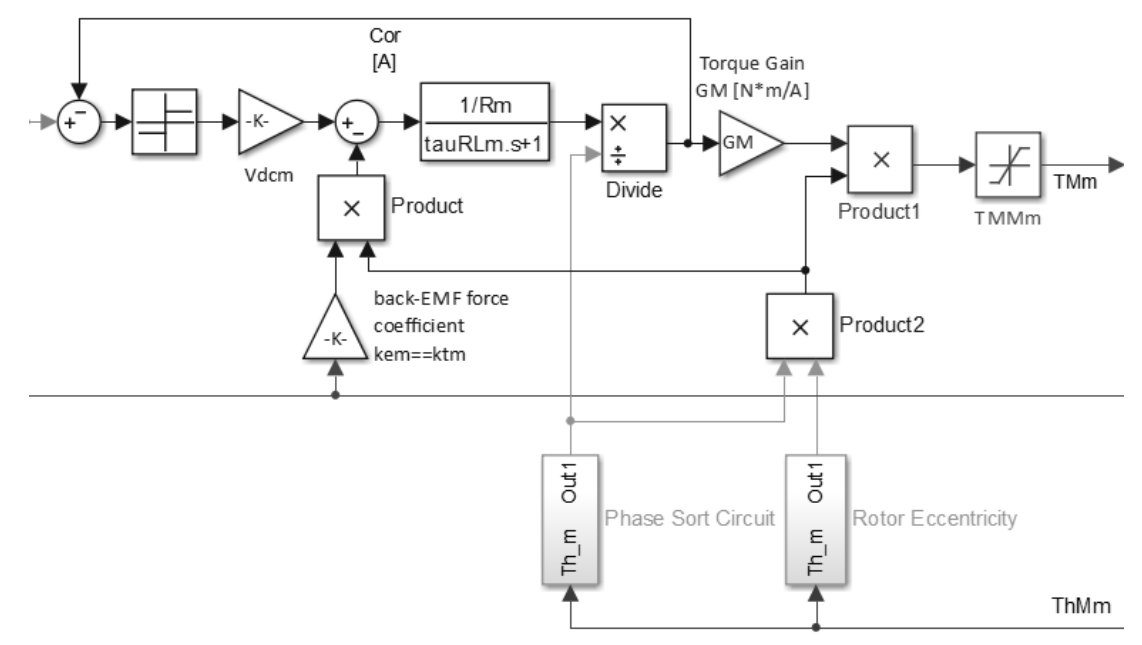

Fig. 5. Schematic of the proposed shape functions (implemented in Matlab-Simulink code) [14] 
Maximum number of generations: Some experimenting have showed that the GA applied to the considered problem usually requires 130 to 180 iterations to achieve convergence; then, the maximum number of allowed generations is increased from the default value of 100 to a conservative 300 , in order to avoid the optimization being stopped before the global minimum is found.

Tolerance on fitness value: The main stopping criterion employed is based on the evaluation of the weighted average change in the fitness function value over generations; if this change is below the function tolerance over the maximum number of stall generations the GA is stopped. A good tradeoff between speed and accuracy is achieved by decreasing the function tolerance from the default 10-6 to 10-12, while keeping the maximum number of stall generations to its default value of 50 .

Parallel computing: A GA optimization requires several evaluations of the fitness function at the same time and each one is independent from the other ones. For this reason, the implementation of parallel computing is straightforward and very advantageous, especially when evaluating an expensive objective function on a multicore processor. According to the Windows Task Manager, the implementation of parallel computing on a quad core Intel i5 3340 processor has taken the processor usage from about $30 \%$ to $100 \%$, reducing computing time to about $1 / 3$.

TABLE I

RESUlTS OF THE PROPOSED FDI METHOD IN CASE OF SINGLE FAULT ISOLATION

\begin{tabular}{|c|c|c|c|}
\hline Single Fault & Test Case $(\boldsymbol{k})$ & $\begin{array}{c}\text { Average } \\
\text { error }\end{array}$ & Variance \\
\hline \multirow{3}{*}{ Dry friction } & {$\left[\begin{array}{llllllllll}0.1 & 0 & 0 & 0 & 0 & 0 & 0.5 & 0.5\end{array}\right]$} & $1.16 \%$ & $5.86 \mathrm{e}-5$ \\
\hline & {$\left[\begin{array}{llllllll}0.4 & 0 & 0 & 0 & 0 & 0 & 0.5 & 0.5\end{array}\right]$} & $1.13 \%$ & $4.73 e-5$ \\
\hline & {$\left[\begin{array}{lllllllll}1.0 & 0 & 0 & 0 & 0 & 0 & 0.5 & 0.5\end{array}\right]$} & $4.54 \%$ & $1.94 \mathrm{e}-3$ \\
\hline \multirow{3}{*}{ Backlash } & {$\left[\begin{array}{llllllll}0 & 0.1 & 0 & 0 & 0 & 0 & 0.5 & 0.5\end{array}\right]$} & $0.61 \%$ & $6.35 e-5$ \\
\hline & {$\left[\begin{array}{llllllll}0 & 0.4 & 0 & 0 & 0 & 0 & 0.5 & 0.5\end{array}\right]$} & $1.72 \%$ & $6.90 \mathrm{e}-4$ \\
\hline & {$\left[\begin{array}{lllllllll}0 & 1.0 & 0 & 0 & 0 & 0 & 0.5 & 0.5\end{array}\right]$} & $1.04 \%$ & $8.57 \mathrm{e}-3$ \\
\hline \multirow{3}{*}{ Short Circuit } & {$\left[\begin{array}{llllllll}0 & 0 & 0.1 & 0 & 0 & 0 & 0.5 & 0.5\end{array}\right]$} & $0.88 \%$ & $3.16 \mathrm{e}-5$ \\
\hline & {$\left[\begin{array}{lllllllll}0 & 0 & 0.2 & 0 & 0 & 0 & 0.5 & 0.5\end{array}\right]$} & $1.30 \%$ & $1.09 \mathrm{e}-4$ \\
\hline & {$\left[\begin{array}{llllllll}0 & 0 & 0.5 & 0 & 0 & 0 & 0.5 & 0.5\end{array}\right]$} & $1.53 \%$ & $8.00 \mathrm{e}-5$ \\
\hline \multirow{3}{*}{ Eccentricity } & {$\left[\begin{array}{llllllll}0 & 0 & 0 & 0 & 0 & 0.1 & 0.5 & 0.5\end{array}\right]$} & $1.39 \%$ & $7.98 \mathrm{e}-4$ \\
\hline & {$\left[\begin{array}{llllllll}0 & 0 & 0 & 0 & 0 & 0.2 & 0.5 & 0.5\end{array}\right]$} & $8.33 \%$ & $3.92 \mathrm{e}-2$ \\
\hline & {$\left[\begin{array}{llllllll}0 & 0 & 0 & 0 & 0 & 0.5 & 0.5 & 0.5\end{array}\right]$} & $3.43 \%$ & $6.66 \mathrm{e}-3$ \\
\hline \multirow{4}{*}{ Gain Drift } & {$\left[\begin{array}{llllllll}0 & 0 & 0 & 0 & 0 & 0 & 0.5 & 0.4\end{array}\right]$} & $0.72 \%$ & $8.54 \mathrm{e}-5$ \\
\hline & {$\left[\begin{array}{lllllllll}0 & 0 & 0 & 0 & 0 & 0 & 0.5 & 0.6\end{array}\right]$} & $1.80 \%$ & $9.26 \mathrm{e}-4$ \\
\hline & {$\left[\begin{array}{lllllllll}0 & 0 & 0 & 0 & 0 & 0 & 0.5 & 0.0\end{array}\right]$} & $3.90 \%$ & $4.15 \mathrm{e}-3$ \\
\hline & {$\left[\begin{array}{llllllll}0 & 0 & 0 & 0 & 0 & 0 & 0.5 & 1.0\end{array}\right]$} & $1.44 \%$ & $7.45 \mathrm{e}-4$ \\
\hline
\end{tabular}

\section{Results}

The proposed FDI prognostic algorithm has been tested in different fault conditions, with single and multiple faults of different magnitude. In order to evaluate the performance of the prognostic technique, a detection error $e_{t o t}$ is defined as:

$$
e_{t o t}=\sqrt{\frac{1}{8}\left(e_{1}^{2}+e_{2}^{2}+e_{3}^{2}+e_{4}^{2}+e_{5}^{2}+e_{6}^{2}+k_{6} e_{7}^{2}+e_{8}^{2}\right)}
$$

which is equivalent to the root mean square error on each variable. It can be noticed that the error on Rotor Eccentricity Phase $e_{7}$ is normalized by multiplying it by the eccentricity amplitude $k_{6}$ : in fact, when eccentricity is small its phase is almost undetermined, and a big error affecting this parameter is irrelevant in terms of fault isolation accuracy.

\section{IV.1. Single Fault Isolation}

For each one of the considered failure modes, three levels of damage are tested to assess the performance of the prognostic technique. Genetic Algorithms are inherently non deterministic, thus different executions with the same initial condition lead to slightly different results and computing times. For this reason, ten optimizations have been performed for each test case, and results are analyzed statistically. Execution time has an average value of $400 \mathrm{~s}$, but a significant variance is observed, being the minimum computing time of $142 \mathrm{~s}$ and the maximum $600 \mathrm{~s}$.

Table I shows the results for those test cases. Results from the optimizations indicate that the algorithm accuracy is independent from the failure mode set in the $\mathrm{RM}$, but is strongly influenced by the magnitude of the fault. In fact, the detection error stands among $1 \%$ to $2 \%$ for low to medium damage levels, while it rises to about $7 \%$ for high damage. This is due to the different fidelity level of the two numerical models, which leads to a slightly different behavior in terms of dynamic response. The error between the two modes diverges for high damage levels, producing a bigger detection error. The case with medium rotor eccentricity, which appears to have a very large detection error, suffers in fact the effect of a single optimization that failed to converge; this is also visible in the higher variance of the results.

However, the main goal of FDI techniques for prognostic purposes is the detection of progressive faults in their incipient state, in order to enable the estimation of Remaining Useful Life (RUL). For this reason, the algorithm is needed to accurately estimate small damage levels, while the accuracy in presence of a heavily damaged system is only of marginal interest.

\section{IV.2. Multiple Faults Isolation}

After the single fault case, the algorithm has been tested also in presence of combinations of different faults. Four test cases are considered, setting in the RM combinations of low, medium and high faults and a combination of medium faults with the addition of signal noise superimposed to the reference current signal.

Table II reports the results obtained in those failure conditions. The multiple fault test cases approximately repeat the results obtained in the single fault conditions: in particular, the detection error is dependent on the fault magnitude and of the same order of the one obtained for the single failure test cases, while the accuracy is approximately the same for all the fault parameters. This 
means that the range of variation of each fault parameter has been chosen coherently with their effect on the measured variable $I_{r e f}$, and the FDI technique has a good robustness in every operating conditions.

As expected, the introduction of a high level of signal noise (in the considered test case a band-limited white noise in the order of $50 \%$ the amplitude of the command signal), the accuracy is comparable to that achieved in the same fault conditions without noise, with a total error in the order of $2 \%$. The high frequency signal components introduced by noise are in fact effectively filtered by inductive effects in the stator coils before affecting the phase current behavior; moreover, the equivalent current signal is further filtered to suppress the effects of the PWM control, which, not being simulated in the MM, would worsen the detection accuracy.

Fig. 6 shows the probability distribution of the detection error, with data gathered in all the performed optimizations. It can be noticed that, for most optimizations, the detection error has a very low value, in the order of $1 \%$. The smaller peak of the probability distribution, settled around $7 \%$, is indeed caused by the simulations executed with a high damage level, which causes the behavior of the two models to diverge slightly. However, this case is not of practical interest for prognostic applications (in fact, such a high damage results in a jammed actuator response and therefore lies in the field of diagnostics), but rather is considered to assess the limits and the applicability of the MM damage implementation. The robustness of the algorithm is also shown by the low variance of the detection error, which means that most of the error is due to the discrepancies between the two models rather than to the nondeterministic nature of the GA.

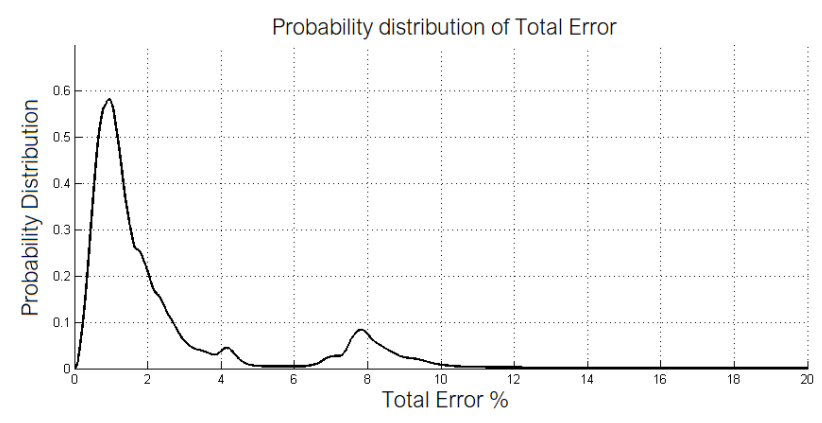

Fig. 6. Probability distribution of the detection error

The performed tests have showed that among the considered failure modes there are no compensating faults, i.e. faults with an opposite effect on the measured variables, which would lead to the risk of false alarms or, which is worse, missed detections. However, the independence of each failure mode shall be investigated as, in future works, other faults are implemented in the models to better represent the behavior of the actual physical EMA, and the prognostic technique will need to be adapted accordingly, for example by monitoring multiple variables for decoupling some failure modes.
TABLE II

RESUlts OF THE PROPOSED FDI METHOD IN CASE OF MULTIPLE FAULT ISOLATION

\begin{tabular}{|c|c|c|c|}
\hline $\begin{array}{l}\text { Multiple Faults } \\
\text { Level }\end{array}$ & Test Case $(\boldsymbol{k})$ & $\begin{array}{c}\text { Average } \\
\text { error }\end{array}$ & Variance \\
\hline Low & {$\left[\begin{array}{llllllllll}0.1 & 0 & 0 & 0 & 0 & 0 & 0.5 & 0.5\end{array}\right]$} & $1.16 \%$ & $5.86 \mathrm{e}-5$ \\
\hline Medium & {$\left[\begin{array}{lllllllll}0 & 0.1 & 0 & 0 & 0 & 0 & 0.5 & 0.5\end{array}\right]$} & $0.61 \%$ & $6.35 e-5$ \\
\hline High & {$\left[\begin{array}{lllllllll}0 & 0 & 0.1 & 0 & 0 & 0 & 0.5 & 0.5\end{array}\right]$} & $0.88 \%$ & $3.16 \mathrm{e}-5$ \\
\hline Medium + Noise & {$\left[\begin{array}{lllllllll}0 & 0 & 0 & 0 & 0 & 0.1 & 0.5 & 0.5\end{array}\right]$} & $1.39 \%$ & $7.98 \mathrm{e}-4$ \\
\hline
\end{tabular}

\section{Conclusion and Future Work}

The study has shown that the use of model based optimizations employing Genetic Algorithms for the prognostic fault detection and identification of Electromechanical Actuators is an effective way to estimate the system health status. These methods combine a great robustness (compared to more traditional optimizations, e.g. gradient-based optimization techniques) with acceptable computing time, especially when the search space has a high dimensionality and therefore the function evaluations will necessarily be very sparse. The on-field use of an algorithm able to detect most common faults in their early stages would allow the actuation of a more effective maintenance program, since each component could be replaced only as needed and almost every corrective intervention could be scheduled in advance. This would yield both a cost reduction, as functional components would not be replaced for having exceeded their design life, and a safety improvement, since faults due to manufacturing flaws which otherwise would cause a premature failure would be isolated as they start to affect the system response and corrected in time.

EMA systems are particularly suitable for the implementation of these tools because most variables are already in the form of digital signals and do not need the introduction of dedicated sensors other than those already present and necessary for the functioning of the control system. Future developments of these prognostic techniques will be aimed to the introduction of prognostics as an integral part of the maintenance procedures. A greater number of faults will be considered and modeled. In particular, failure modes of the sensors and control electronics will be introduced with higher detail, along with the demagnetization of rotor permanent magnets.

Additionally, the computing performances will be improved by further simplifying the Monitor Model, or turning it into a non-physical surrogate model able to be computed in a much shorter time, while keeping an acceptable accuracy in reproducing the behavior of the RM. The algorithm will be assessed with different test commands and load profiles in order to allow in-flight FDI.

\section{References}

[1] R. E. J. Quigley, More Electric Aircraft, Proceedings of the Eighth Annual IEEE Applied Power Electronics Conference (APEC' '93), 906-911 (1993). 
[2] M. Howse, All-Electric Aircraft, Power Engineer (2003), 17(4):35-37.

[3] A. G. Garriga, S.S. Ponnusamy, L. Mainini, A multi-fidelity framework to support the design of More-Electric Actuation, 2018 Multidisciplinary Analysis and Optimization Conference, AIAA Aviation Forum, (AIAA Paper 2018-3741), American Institute of Aeronautics and Astronautics, Atlanta, USA (2018).

[4] Ismagilov, F., Vavilov, V., Tarasov, N., Ayguzina, V., HighTemperature Starter-Generator with Fractional-Slot Concentrated Windings for More Electric Aircraft: Design and Testing of the Scaled-Size Prototype, (2017) International Review of Aerospace Engineering (IREASE), 10 (2), pp. 81-89. doi:https://doi.org/10.15866/irease.v10i2.12038

[5] Y. Hussain, S. Burrow, P. Keogh, L. Henson, A Review of Techniques to Mitigate Jamming in Electromechanical Actuators for Safety Critical Applications, International Journal of Prognostics and Health Management, (2018), 9.

[6] F.L.J. Linden, N. Dreyer, A. Dorkel, EMA Health Monitoring: An overview, Recent Advances in Aerospace Actuation Systems and Components, (2016), 21-26.

[7] Chin, C., Abdullah, S., Singh, S., Ariffin, A., Establishing Energy-Damage Relationship for Fatigue Data Editing of Strain Loading History, (2018) International Review of Mechanical Engineering (IREME), 12 (7), pp. 612-619. doi:https://doi.org/10.15866/ireme.v12i7.14426

[8] Zahiri, L., Mighouar, Z., Khatib, H., Mansouri, K., Salhi, B., Fatigue Life Analysis of Dented Pipes Subjected to Internal Pressure, (2017) International Review of Mechanical Engineering (IREME), 11 (8), pp. 587-596. doi:https://doi.org/10.15866/ireme.v11i8.12089

[9] Purnowidodo, A., Chairul Anam, M., Subekti, D., The Effect of Stress Amplitude and Ratio on Crack Growth Behaviour in Fibre Metal Laminate, (2017) International Review of Mechanical Engineering (IREME), 11 (11), pp. 883-891. doi:https://doi.org/10.15866/ireme.v11i11.12634

[10] Deshmukh, A., Venkatachalam, G., Saraf, M., Fatigue Life Prediction of Doubler Plate Weld Joint Using Virtual Strain Gauge, Validation through Experiments and Its Application, (2018) International Review of Mechanical Engineering (IREME), 12 (2), pp. 196-206. doi:https://doi.org/10.15866/ireme.v12i2.14625

[11] G. Vachtsevanos, F. Lewis, M. Roemer, A. Hess, B. Wu, Intelligent Fault Diagnosis and Prognosis for Engineering Systems (Wiley, 2006).

[12] C.S. Byington, W. Watson, D. Edwards, P. Stoelting, A ModelBased Approach to Prognostics and Health Management for Flight Control Actuators, IEEE Aerospace Conference Proceedings, Big Sky, MT, USA. (2004).

[13] Santos, L., Melicio, R., Stress, Pressure and Fatigue on Aircraft Maintenance Personal, (2019) International Review of Aerospace Engineering (IREASE), 12 (1), pp. 35-45. doi:https://doi.org/10.15866/irease.v12i1.14860

[14] P.C. Berri, M.D.L. Dalla Vedova, P. Maggiore, A Smart Electromechanical Actuator Monitor for New Model-Based Prognostic Algorithms, International Journal of Mechanics and Control (JoMaC). (2016), 17(2):59-66.

[15] L. Borello, M.D.L. Dalla Vedova, G. Jacazio, M. Sorli, A Prognostic Model for Electrohydraulic Servovalves, Proceedings of the 2009 Annual Conference of the Prognostics and Health Management Society (PHM 2009), San Diego, CA (2009).

[16] A. Raie, V. Rashtchi, Using a genetic algorithm for detection and magnitude determination of turn faults in an induction motor, Electrical Engineering (2002), 84(5):275-279.

[17] M. Alamyal, S.M. Gadoue, B. Zahawi, Detection of induction machine winding faults using genetic algorithm. Diagnostics for Electric Machines, Power Electronics and Drives (SDEMPED), 9th IEEE International Symposium, Valencia, Spain, 157-161 (2013).

[18] P.C. Berri, M.D.L. Dalla Vedova, P. Maggiore, On-board electromechanical servomechanisms affected by progressive faults: proposal of a smart GA model-based prognostic approach, Proceedings of the 27th European Safety and Reliability Conference (ESREL 2017), Portoroz, Slovenia. 839-845 (2017).

[19] M.D.L. Dalla Vedova, P. Maggiore, L. Pace, A New Prognostic
Method Based on Simulated Annealing Algorithm to Deal with the Effects of Dry Friction on Electromechanical Actuators, International Journal of Mechanics (2015), 9:236-245.

[20] Kabashi, Q., Limani, M., Caka, N., Zabeli, M., Low Order Harmonic Analysis of 3-Phase SPWM and SV-PWM Inverter Systems Driving an Unbalanced 3-Phase Induction Motor Load, (2018) International Review on Modelling and Simulations (IREMOS), 11 (3), pp. 134-142. doi:https://doi.org/10.15866/iremos.v11i3.14586

[21] El Mekki, A., Ben Saad, K., Discrete and Parametric Fault Diagnosis of an Inverter-Driven Brushless DC Motor Using a Hybrid Formalism, (2018) International Review of Automatic Control (IREACO), 11 (5), pp. 226-233. doi:https://doi.org/10.15866/ireaco.v11i5.14781

[22] P.C. Berri. M.D.L. Dalla Vedova, P. Maggiore, Enhanced hybrid prognostic approach applied to aircraft on-board electromechanical actuators affected by progressive faults, Proc. of the 28th European Safety and Reliability Conference (ESREL 2018), Trondheim, Norway (2018), 1077-1084.

[23] M.D.L. Dalla Vedova, P.C. Berri, S. Re, Metaheuristic BioInspired Algorithms for Prognostics: Application to On-Board Electromechanical Actuators, Proceedings of the 3rd International Conference on System Reliability and Safety (ICSRS 2018), Barcelona, Spain (2018).

[24] Belmonte, D., Dalla Vedova, M., Maggiore, P., Prognostics of Onboard Electromechanical Actuators: a New Approach Based on Spectral Analysis Techniques, (2018) International Review of Aerospace Engineering (IREASE), 11 (3), pp. 96-103. doi:https://doi.org/10.15866/irease.v11i3.13796

[25] Bindu, S., Thomas, V., A Modified Direct-Quadrature Axis Model for Characterization of Air-gap Mixed Eccentricity Faults in Three-Phase Induction Motor, (2018) International Review on Modelling and Simulations (IREMOS), 11 (6), pp. 359-365. doi:https://doi.org/10.15866/iremos.v11i6.15513

[26] Khodja, M., Boudinar, A., Bendiabdellah, A., Effect of Kaiser Window Shape Parameter for the Enhancement of Rotor Faults Diagnosis, (2017) International Review of Automatic Control (IREACO), 10 (6), pp. 461-467. doi:https://doi.org/10.15866/ireaco.v10i6.13077

[27] Kouadria, M., Boudinar, A., Bendiabdellah, A., Benouzza, N., Induction Motor Stator Fault Diagnosis by Rotor Slots Harmonics Tracking Using Prony Improved Approach, (2017) International Review of Automatic Control (IREACO), 10 (4), pp. 296-305. doi:https://doi.org/10.15866/ireaco.v10i4.11880

[28] El Yousfi Alaoui, M., Jilbab, A., El Hani, S., FPGA Design and Implementation of an Optimized Adaptive Filter for Real Time Extraction of Vibration Signal Related to Bearing Defects, (2016) International Review on Modelling and Simulations (IREMOS), 9 (2), pp. 105-113. doi:https://doi.org/10.15866/iremos.v9i2.8009

[29] Sadiki, L., El Hani, S., Guedira, S., Ouachtouk, I., Assessment of Time Frequency Color Index for Electrical Machines Diagnosis and Fault Severity, (2016) International Review on Modelling and Simulations (IREMOS), 9 (1), pp. 1-10. doi:https://doi.org/10.15866/iremos.v9i1.7613

[30] M.S. Mamis, M. Arkan, C. Keles, Transmission lines fault location using transient signal spectrum, International Journal of Electrical Power and Energy Systems (2013), 714-718

[31] M.D.L. Dalla Vedova, P. Maggiore, L. Pace. Proposal of Prognostic Parametric Method Applied to an Electrohydraulic Servomechanism Affected by Multiple Failures, WSEAS Transactions on Environment and Development (2014), 10:478490.

[32] H. Su, K.T, Chong, Induction machine condition monitoring using neural network modelling, IEEE Transactions on Industrial Electronics. (2007) 54(1):241-249.

[33] S. Hamdani, O. Touhami, R. Ibtiouen, M. Fadel, Neural network technique for induction motor rotor faults classification-dynamic eccentricity and broken bar faults, IEEE International Symposium on Diagnostics for Electric Machines, Power Electronics \& Drives. (2011), 626-631.

[34] S.S. Refaat, H. Abu-Rub, M.S. Saad, E.M. Aboul-Zahab, A. Iqbal, ANN-based for detection, diagnosis the bearing fault for three phase induction motors using current signal. IEEE 
International Conference on Industrial Technology (ICIT), 253258 (2013).

[35] M.D.L. Dalla Vedova, D. De Fano, P. Maggiore, Neural Network Design for Incipient Failure Detection on Aircraft EM Actuator, International Journal of Mechanics and Control (JoMaC) (2016), 17(1):77-83.

[36] I. Chopra, R. Ganguli, D.J. Haas, Detection of Helicopter Rotor System Simulated Faults Using Neural Networks, Journal of the American Helicopter Society (1997) 42:161-171.

[37] Menasria, Y., Debbache, N., A Robust Actuator Fault Detection and Isolation Approach for Nonlinear Dynamic Systems, (2016) International Journal on Numerical and Analytical Methods in Engineering (IRENA), 4 (3), pp. 83-89.

[38] Taha, I., Ghoneim, S., Zaini, H., A Fuzzy Diagnostic System for Incipient Transformer Faults Based on DGA of the Insulating Transformer Oils, (2016) International Review of Electrical Engineering (IREE), 11 (3), pp. 305-313. doi:https://doi.org/10.15866/iree.v11i3.8453

[39] M.D.L. Dalla Vedova, P. Maggiore, L. Pace, A. Desando, Evaluation of the correlation coefficient as a prognostic indicator for electromechanical servomechanism failures, International Journal of Prognostics and Health Management (2015) 6(1).

[40] Soufi, Y., Bahi, T., Harkat, M., Mohammedi, M., Fault Diagnosis Methods for Three Phase PWM Inverter Fed Induction Motor, (2018) International Journal on Engineering Applications (IREA), 6 (4), pp. 122-127.

doi:https://doi.org/10.15866/irea.v6i4.16022

[41] Sawitri, D., Heryanto, M., Suprijono, H., Purnomo, M., Kusumoputro, B., Vibration-Signature-Based Inter-Turn Short Circuit Identification in a Three-Phase Induction Motor Using Multiple Hidden Layer Back Propagation Neural Networks, (2018) International Review of Electrical Engineering (IREE), 13 (2), pp. 98-106. doi:https://doi.org/10.15866/iree.v13i2.13881

[42] Azouzi, K., Boudinar, A., Bendiabdellah, A., Detection of Bearing Faults in Induction Motor by a Combined Approach SVD-Kalman Filter, (2018) International Review of Automatic Control (IREACO), 11 (1), pp. 14-22. doi:https://doi.org/10.15866/ireaco.v11i1.13501

[43] P.C. Berri, M.D.L. Dalla Vedova, L. Mainini, Real-time Fault Detection and Prognostics for Aircraft Actuation Systems, AIAA Scitech 2019 Forum, (AIAA Paper 2019-2210), American Institute of Aeronautics and Astronautics, San Diego, California, USA (2019).

[44] M.D.L. Dalla Vedova, P. Maggiore, L. Pace, A new prognostic method based on simulated annealing algorithm to deal with the effects of dry friction on electromechanical actuators. International Journal of Mechanics (2015), 9:236-245.

[45] D. Belmonte, M.D.L. Dalla Vedova, P. Maggiore, Electromechanical servomechanisms affected by motor static eccentricity: Proposal of fault evaluation algorithm based on spectral analysis techniques. Safety and Reliability of Complex Engineered Systems - Proceedings of the 25th European Safety and Reliability Conference (ESREL 2015), 2365-2372 (2015).

[46] T.A. Haskew, D.E. Schinstock, E. Waldrep, Two-Phase On Drive Operation in a Permanent Magnet Synchronous Machine Electromechanical Actuator, IEEE Transactions on Energy Conversion (1999), 14.

[47] B.K. Lee, M. Ehsani, Advanced Simulation Model for Brushless DC Motor Drives, Electric Power Components and Systems (2003), 31(9): 841-868.

[48] T. Hemanand, T. Rajesh, Speed Control of Brushless DC Motor Drive Employing Hard Chopping PWM Technique Using DSP. Proceedings of India International Conference on Power Electronics (2006).

[49] J. Hua, H. Zhiyong, Simulation of Sensorless Permanent Magnetic Brushless DC Motor Control System, Proceedings of the IEEE International Conference on Automation and Logistics, China (2008).

[50] A. Halvaei Niasar, H. Moghbelli, A. Vahedi, Modelling, Simulation and Implementation of Four-Switch Brushless DC Motor Drive Based On Switching Functions, IEEE EUROCON 2009, St. Petersburg, Russia, 682 -687 (2009).
[51] M. Çunkas, O. Aydoğdu, Realization of Fuzzy Logic Controlled Brushless DC Motor Drives using Matlab/Simulink. Mathematical and Computational Applications (2010) 15:218229.

[52] L. Borello, G. Villero, M.D.L. Dalla Vedova, New asymmetry monitoring techniques: effects on attitude control, Aerospace Science and Technology (2009), 13(8): 475-487.

[53] L. Borello, G. Villero, M.D.L. Dalla Vedova, Flaps Failure and Aircraft Controllability: Developments in Asymmetry Monitoring Techniques, Journal of Mechanical Science and Technology, (2014) 28(11):4593-4603.

[54] L. Borello, M.D.L. Dalla Vedova, A dry friction model and robust computational algorithm for reversible or irreversible motion transmission, International Journal of Mechanics and Control (2012) 13(2):37-44.

[55] T. Kenjo, S. Nagamori, Brushless Motors: Advanced Theory and Modern Applications, third ed. (Sogo Electronics Press, Tokio, Japan, 2003).

[56] J.C. Chesley, Handbook of Reliability Prediction Procedures for Mechanical Equipment (USA Navy, Naval Surface Warfare Center, NSWC Carderock Division, 2011).

[57] J. Weiss, Control Actuation Reliability and Redundancy for Long Duration, Underwater Vehicle Missions with High Value Payloads, Proceedings of the 2014 Underwater Intervention Conference (UI 2014), New Orleans, Louisiana, USA (2014).

[58] Mohite, M., Kothavale, B., Experimental and Analytical Analysis of Crack Propagation on Spur Gear Due to Bending Fatigue and Service Life Estimation, (2018) International Review of Mechanical Engineering (IREME), 12 (1), pp. 88-96. doi:https://doi.org/10.15866/ireme.v12i1.14140

[59] I. Markovsky, S. Van Huffel. Overview of total least-squares methods, Signal Processing (2007), 87(10):2283-2302.

\section{Authors' information}

Dept. of Mechanical and Aerospace Engineering (DIMEAS), Politecnico di Torino (Italy).

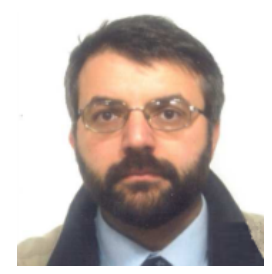

Matteo D. L. Dalla Vedova (Savona, Italy, 1975) received his M.Sc. and the Ph.D. in aerospace engineering at Politecnico di Torino in 2003 and 2007, respectively. He is currently Assistant Professor in the Department of Mechanics and Aerospace Engineering (DIMEAS). His research activity is mainly focused on the aeronautical systems engineering and, in particular, is dedicated to design, analysis and numerical simulation of on board systems, study of secondary flight control systems and conception of related monitoring strategies, development of prognostic algorithms for aerospace servomechanism and study of innovative primary flight control architectures.

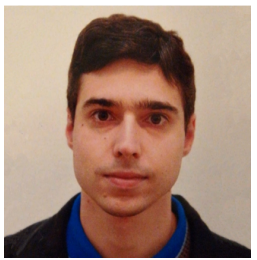

Pier Carlo Berri (Tortona, Italy, 1992) received his Bs.C. and Ms.C. degrees in aerospace engineering at Politecnico di Torino (Torino, Italy) in 2014 and 2016. He is currently a Ph.D. student in aerospace engineering at Politecnico di Torino. His research activity is focused on prognostics and diagnostics of aerospace systems, electro-mechanical actuation systems and minimally intrusive sensors for aerospace applications. Mr. Berri is a student member of the American Institute of Aeronautics and Astronautics (AIAA) since 2018. 